\title{
CURVATURE AND CHARACTERISTIC CLASSES OF COMPACT RIEMANNIAN MANIFOLDS
}

\author{
YUK-KEUNG CHEUNG \& CHUAN-CHIH HSIUNG \\ In memory of Professor Vernon G. Grove
}

\section{Introduction}

During the past quarter century the development of the theory of fibre bundles has led to a new direction in differential geometry for studying relationships between curvatures and certain topological invariants such as characteristic classes of a compact Riemannian manifold. Along this direction the first and simplest result is the Gauss-Bonnet formula [2], [3], which expresses the Euler-Poincaré characteristic of a compact orientable Riemannian manifold of even dimension $n$ as an integral of the $n$-th sectional curvature or the Lipschitz-Killing curvature times the element of area of the manifold. Later, Chern [5] obtained curvature conditions respectively for determining the sign of the Euler-Poincaré characteristic and for the vanishing of the Pontrjagin classes of a compact orientable Riemannian manifold. Recently, Thorpe [8] extended a special case of Chern's conditions by using higher order sectional curvatures, which are weaker invariants of the Riemannian structure than the usual sectional curvature. The purpose of this paper is to further extend the conditions of both Chern and Thorpe.

In $\S 1$, for a Riemannian manifold the equations of structure are given, and higher order sectional curvatures and related differential forms are defined. $\S 2$ contains the differential forms expressing, respectively, the Euler-Poincaré characteristic and the Pontrjagin classes of compact orientable Riemannian manifolds in the sense of de Rham's theorem. In $\S 3$, we first define some general curvature conditions, and then use them to extend the above mentioned results of Chern and Thorpe. The proofs of the main results (Theorems 3.1 and 3.2) of this paper are easily deduced from several lemmas.

\section{Higher order sectional curvatures}

Let $M$ be a Riemannian manifold of dimension $n$ (and class $C^{\infty}$ ), and $V_{x}, V_{x}^{*}$ respectively the spaces of tangent vectors and covectors at a point $x$ of the manifold $M$. By taking an orthonormal basis in $V_{x}$ and its dual basis in $V_{x}^{*}$, over a neighborhood $U$ of the point $x$ on the manifold $M$, we then have a family of orthonormal frames $x e_{1} \cdots e_{n}$

Communicated April 20, 1967. The work of the second author was partially supported by the National Science Foundation grant GP-4222. 
and linear differential forms $\omega_{1}, \cdots, \omega_{n}$ such that $\left\langle e_{i}, \omega_{j}\right\rangle=\delta_{i j}(=1$ for $i=j$, and $=0$ otherwise), and the Riemann metric is of the form

$$
d s^{2}=\sum_{i} \omega_{i}^{2}
$$

Throughout this paper all Latin indices take the values $1, \cdots, n$ unless stated otherwise. The equations of structure of the Riemann metric are

$$
\begin{aligned}
d \omega_{i} & =\sum_{j} \omega_{j} \wedge \omega_{j i}, & \omega_{i j}+\omega_{j i}=0, \\
d \omega_{i j} & =\sum_{k} \omega_{i k} \wedge \omega_{k j}+\Omega_{i j}, & \Omega_{i j}+\Omega_{j i}=0,
\end{aligned}
$$

and the Bianchi identities are

$$
\begin{aligned}
& \sum_{j} \omega_{j} \wedge \Omega_{j i}=0 \\
& d \Omega_{i j}+\sum_{k} \Omega_{i k} \wedge \omega_{k j}-\sum_{k} \omega_{i k} \wedge \Omega_{k j}=0
\end{aligned}
$$

where the wedge $\wedge$ denotes the exterior multiplication.

In terms of a local coordinate system $u^{1}, \cdots, u^{n}$ in the neighborhood $U$ let

$$
e_{i}=\sum_{k} X_{i}^{k} \partial / \partial u^{k}
$$

Then

$$
\Omega_{i j}=\frac{1}{2} \sum_{k, l} S_{i j k l} \omega_{k} \wedge \omega_{l}
$$

where

$$
S_{i j k l}=R_{p q r s} X_{i}^{p} X_{j}^{q} X_{k}^{r} X_{l}^{s},
$$

repeated indices implying summation over their ranges, and $R_{p q r s}$ being the Riemann-Christoffel tensor.

Throughout this paper, for indices we shall use $I(p)$ to indicate the ordered set of $p$ integers $i_{1}, \cdots, i_{p}$ among $1, \cdots, n$. When more than one set of indices is needed at one time, we shall use other capital letters such as $J, H, R, S, \cdots$ in addition to $I$. Now for an even $p \leq n$, we define the following $p$-form:

$$
\Theta_{I(p)}=\frac{1}{p !} \delta_{I(p)}^{J(p)} \Omega_{j_{1} j_{2}} \wedge \cdots \wedge \Omega_{j p-1 j p}
$$

where $\delta_{I(p)}^{J(p)}$ is +1 (respectively -1$)$, if the integers $i_{1}, \cdots, i_{p}$ are distinct and $J(p)$ is an even (respectively odd) permutation of $I(p)$; it is zero in all other cases. Clearly, $\Theta_{i j}=\Omega_{i j}$. These forms $\Theta_{I(p)}$, except for constant factors, were first used by Chern in [3], [5]. For an even $n$, $\Theta_{1 \ldots n}$ is intrinsic and called the Gauss curvature form of the manifold $M$, and the $p$-th Gauss curvature form studied by Eells [6] is closely 
related to $\Theta_{1} \ldots p$. By using equation (1.5), equation (1.7) can be written in the form

$$
\Theta_{I(p)}=\frac{1}{2^{p / 2} p !} \sum_{H(p)} \delta_{I(p)}^{J(p)} S_{j_{1} j_{2} h_{1} h_{2}} \cdots S_{j p-1 j p^{h} p-1^{h} p} \omega_{H(p)}
$$

where we have placed

$$
\omega_{H(p)}=\omega_{h_{1}} \wedge \cdots \wedge \omega_{h_{p}}
$$

For each $p$-dimensional plane $P$ in the tangent space $V_{x}$ of the manifold $M$ at a point $x$, the Lipschitz-Killing curvature at the point $x$ of the $p$-dimensional geodesic submanifold of the manifold $M$ tangent to $P$ at the point $x$ is called the $p$-th sectional curvature of the manifold $M$ at the point $x$ with respect to the $p$-dimensional $P$, and is given (see for instance [1, p. 257]) in terms of any orthonormal basis $e_{i_{1}}, \cdots, e_{i_{p}}$ of $P$ by

$$
\begin{gathered}
K_{I(p)}(P)=\frac{(-1)^{p / 2}}{2^{p / 2} p !} \delta_{J(p)}^{R(p)} \delta_{H(p)}^{S(p)} R_{r_{1} r_{2} s_{1} s_{2}} \cdots R_{r p-1^{r} p^{s} p-1^{s} p} \\
\cdot X_{i_{1}}^{j_{1}} \cdots X_{i_{p}}^{j_{p}} X_{i_{1}}^{h_{1}} \cdots X_{i_{p}}^{h_{p}}
\end{gathered}
$$

From the geometric structure it is obvious that $K_{I(p)}(P)$ is independent of the choice of the orthonormal basis $e_{i_{1}}, \cdots, e_{i_{p}}$ of $P$. For $p=2, K_{I(p)},(P)$ is the usual Riemannian sectional curvature of the manifold $M$ at the point $x$ with respect to the plane $P$, and for $p=n$ (even), it is the Lipschitz-Killing curvature of the manifold $M$ at the point $x$. By using equation (1.6), equation (1.10) is readily reduced to

$$
K_{I(p)}(p)=\frac{(-1)^{p / 2}}{2^{p / 2} p !} \delta_{I(p)}^{J(p)} \delta_{I(p)}^{H(p)} S_{j_{1} j_{2} h_{1} h_{2}} \cdots S_{j_{p-1} j p^{h} p-1^{h} p}
$$

\section{Characteristic classes}

Let $V$ be a vector space of dimension $n$ over the real field $R$, and $V^{*}$ its dual space. Then there is a pairing of $V$ and $V^{*}$ into $R$, which we denote by $<X, X^{\prime}>\in R, X \in V, X^{\prime} \in V^{*}$. The Grassmann algebra of $\wedge(V)$ of $V$ is a graded algebra admitting a direct sum decomposition

$$
\wedge(V)=\wedge^{0}(V)+\wedge^{1}(V)+\cdots+\wedge^{n}(V)
$$

where $\wedge^{r}(V)$ is the subspace of all homogeneous elements of $\wedge(V)$ of degree $r$. From $\wedge(V)$ and the Grassmann algebra $\wedge\left(V^{*}\right)$ of $V^{*}$ we from their tensor product $\wedge(V) \otimes \wedge\left(V^{*}\right)$, which is generated as a vector space by products of the form $\xi \otimes \xi^{\prime}, \xi \in \wedge(V), \xi^{\prime} \in \wedge\left(V^{*}\right)$. It should be remarked that if $\xi^{\prime} \in \wedge\left(V^{*}\right), \eta \in \wedge(V), \xi \in \wedge(V), \eta^{\prime} \in \wedge\left(V^{*}\right)$, then 


$$
\left(\xi \otimes \xi^{\prime}\right) \wedge\left(\eta \otimes \eta^{\prime}\right)=(\xi \wedge \eta) \otimes\left(\xi^{\prime} \wedge \eta^{\prime}\right)
$$

Suppose now a scalar product be given in $V$. We will be interested in the subspace $\wedge^{2 k}(V) \otimes \wedge^{2 k}\left(V^{*}\right)$ of $\wedge(V) \otimes \wedge\left(V^{*}\right)$. If $e_{1}, \cdots, e_{n}$ form an orthonormal basis of $V$, the elements $e_{i_{1}} \wedge \cdots \wedge e_{i_{2 k}}$, for all combinations of $i_{1}, \cdots, i_{2 k}$ among $1, \cdots, n$, constitute an othonormal basis of $\wedge^{2 k}(V)$, and an element $A$ of $\wedge^{2 k}(V) \otimes \wedge^{2 k}\left(V^{*}\right)$ can be written in the form

$$
A=\sum_{(i)}\left(e_{i_{1}} \wedge \cdots \wedge e_{i_{2 k}}\right) \otimes \xi_{l(2 k)}^{\prime}
$$

where $\xi_{l(2 k)}^{\prime} \in \wedge^{2 k}\left(V^{*}\right)$, and $\sum_{(i)}$ denotes the summation over all the different combinations of $i_{1}, \cdots, i_{2 k}$ among $1, \cdots, n$. We call

$$
|A|^{2}=\sum_{(i)} \xi_{l(2 k)}^{\prime 2} \in \wedge^{4 k}\left(V^{*}\right)
$$

the square of the measure of A; it is clearly independent of the orthonormal basis $e_{1}, \cdots, e_{n}$.

These algebraic notions can be applied naturally to the space $V_{x}$ of tangent vectors and the space $V_{x}^{*}$ of covectors at a point $x$ of a differentiable manifold $M$ of dimension $n$ (and class $C^{\infty}$ ). Suppose that the equations of structure of the Riemann metric on the manifold $M$ be given by equations (1.2). Then introduce

$$
\Omega=\sum_{i, j}\left(e_{i} \wedge e_{j}\right) \otimes \Omega_{i j}
$$

For an even positive integer $p \leq n$, we can easily find

$$
\Omega^{p / 2}=\sum_{(i)}\left[\left(e_{i_{1}} \wedge \cdots \wedge e_{i_{p}}\right) \otimes\left(\Omega_{i_{1} i_{2}} \wedge \cdots \wedge \Omega_{i_{p-1} i_{p}}\right)\right] .
$$

For a fixed set $I(p)$, from equations $(2.6),(1.7)$ it follows that the coefficient of the term $e_{i_{1}} \wedge \cdots \wedge e_{i_{p}}$ in $\Omega^{p / 2}$ is equal to $p$ ! $\Theta_{I(p)}$, so that

$$
\Omega^{p / 2}=p ! \sum_{(i)}\left[\left(e_{i_{1}} \wedge \cdots \wedge e_{i_{p}}\right) \otimes \Theta_{I(p)}\right] .
$$

In particular, for an even $n$, equation (2.7) becomes

$$
\Omega^{n / 2}=n !\left(e_{1} \wedge \cdots \wedge e_{n}\right) \otimes \Theta_{1 \cdots n}
$$

Thus we can formulate the Gauss-Bonnet formulas as follows (see for instance [3]): 
Theorem 2.1. Let $M$ be a compact orientable Riemannian manifold of even dimension $n$. Then the Euler-Poincaré characteristic $\chi(M)$ of $M$ is given by the inetgral

$$
\chi(M)=\frac{(-1)^{n / 2} n !}{2^{n} \pi^{n / 2}(n / 2) !} \int_{M} \Theta_{1 \cdots n}
$$

From equations $(2.7),(2.4)$ we obtain immediately

$$
\left|\Omega^{k}\right|^{2}=[(2 k) !]^{2} \sum_{(i)}\left(\Theta_{I(2 k)}\right)^{2}
$$

and therefore a theorem of Chern [5] can be stated in the following form:

Theorem 2.2. The differential form

$$
\frac{[2 k) !]^{2}}{\left(2^{k} k !\right)^{2}(2 \pi)^{2 k}} \sum_{(i)} \Theta_{I(2 k)} \wedge \Theta_{I(2 k)}
$$

defines the $k$-th Pontrjagin class ${ }^{1} P_{k}$ of a compact orientable Riemannian manifold in the sense of de Rham's theorem.

\section{Relationships between curvatures and characteristic classes}

Let $M$ be a Riemannian manifold of dimension $n, p$ an even positive integer $\leq n$, and $a_{i h}=a_{h i}, i, h=1, \cdots, n$, given smooth real-valued functions on the manifold $M$ at a point $x$. Denote

$$
A_{I(p) . H(p)}=\left|a_{i_{\alpha} h_{\beta}}\right| \quad(\alpha, \beta=1, \cdots, p),
$$

where the rows and columns of the determinant $\left|a_{i_{\alpha} h_{\beta}}\right|$ are arranged in the natural order of $\alpha$ and $\beta$, respectively. At the point $x$ on the manifold $M$ we then consider the following curvature condition:

$$
\delta_{I(p)}^{I(p)} S_{j_{1} j_{2} h_{1} h_{2}} \cdots S_{j_{p-1} j_{p} h_{p-1} h_{p}}=2^{p / 2} k_{p} A_{I(p) . H(p)}
$$

for all $I(p), H(p) \in(1, \cdots, n)$, where $k_{p}$ is a smooth real-valued function on the manifold $M$ at the point $x$. For $p=2$, this condition has been used by Chern in [5]. From equations (3.2), (1.8), (1.9), (1.11) follows immediately

Lemma 3.1. For a fixed set of indices $I(p)$ condition (3.2) implies

$$
\begin{aligned}
& \Theta_{I(p)}=\frac{1}{p !} k_{p} \sum_{H(p)} A_{I(p) \cdot H(p)} \omega_{H(p)}, \\
& K_{I(p)}=(-1)^{p / 2} k_{p} A_{I(p), I(p)},
\end{aligned}
$$

and also equation (3.3) implies condition (3.2).

\footnotetext{
${ }^{1}$ For the definition see also:[4].
} 
In particular, when $a_{i j}=\delta_{i j}$, then

$$
A_{I(p), H(p)}=\Delta_{I(p), H(p)},
$$

where

$$
\Delta_{I(p), H(p)}=\left|\delta_{i \alpha h_{\beta}}\right| \quad(\alpha, \beta=1, \cdots, p),
$$

and therefore equations (3.3), (3.4) are reduced to

$$
\begin{aligned}
& \Theta_{I(p)}=\kappa_{p} \omega_{I(p)}, \\
& K_{I(p)}=(-1)^{p / 2} \kappa_{p} .
\end{aligned}
$$

Thus, from equation (3.8) we have

Lemma 3.2. Condition (3.2) with $a_{i j}=\delta_{i j}$ implies that the $p$-th sectional curvature $K_{I(p)}(P)$ at the point $x$ of the manifold $M$ is constant, that is, independent of the p-dimensional plane $P$ at the point $x$.

On the other hand, from equations (1.8), (1.11) it follows immediately that equation (3.7) implies equation (3.8). The converse is also true as was proved by Thorpe [8], so that we can state, altogether,

Lemma 3.3. Equations (3.7) and (3.8) are equivalent.

For the converse of Lemma 3.2, we notice that $[7$, p. 238]

$$
\delta_{I(p), H(p)} \Delta_{I(p), H(p)},
$$

where $\delta_{I(p), H(p)}$ is defined exactly in the same way as $\delta_{I(p)}^{H(p)}$, so that equation (3.7) can be written as

$$
\begin{aligned}
\Theta_{I(p)} & =\frac{1}{p !} \kappa_{p} \sum_{H(p)} \delta_{I(p), H(p)} \omega_{H(p)} \\
& =\frac{1}{p !} \kappa_{p} \sum_{H(p)} \Delta_{I(p), H(p)} \omega_{H(p)} .
\end{aligned}
$$

A comparison of equation (3.10) with equation (1.8) yields immediately condition (3.2) with $a_{i j}=\delta_{i j}$. By using Lemma 3.3 and combining the result with Lemma 3.2 we hence obtain

Lemma 3.4. The $p$-th sectional curvature $K_{I(p)}$ of the manifold $M$ at a point $x$ is constant if and only if condition (3.2) with $a_{i j}=\delta_{i j}$ holds.

Lemma 3.5. On a Riemannian manifold $M$ of dimension $n$, if condition (3.2) holds for some even $p$ and $q$ with $p+q \leq n$, then

$$
\Theta_{I(p+q)}=\frac{1}{(p+q) !} \kappa_{p} \kappa_{q} \sum_{H(p+q)} A_{I(p+q), H(p+q)} \omega_{H(p+q)},
$$

so that condition (3.2) also holds for $p+q$ with $\kappa_{p+q}=\kappa_{p} \kappa_{q}$. 
Proof of Lemma 3.5. Let the set $I(p+q)$ have distinct elements, and $\left(I_{1}(p), I_{2}(q)\right)$ be a partition of $I(p+q)$, where $I_{1}(p)=\left(i_{11}, \cdots, i_{1 p}\right)$ and $I_{2}(q)=\left(i_{21}, \cdots, i_{2 q}\right)$. Then, from equation (1.7),

$$
\begin{gathered}
\Theta_{I(p+q)}=\frac{1}{(p+q) !} \sum_{\left(I_{1}, I_{2}\right)} \delta_{I(p+q)}^{I_{1}(p) I_{2}(q)} \Omega_{i_{11} i_{12}} \wedge \cdots \wedge \Omega_{i_{1, p-1} i_{1 p}} \\
\wedge \Omega_{i_{21} i_{22}} \wedge \cdots \wedge \Omega_{i_{2 . q-1} i_{2 q}}
\end{gathered}
$$

where $\sum_{\left(I_{1}, I_{2}\right)}$ denotes the summation over all such partitions of $I(p+$ $q)$ into $\left(I_{1}(p), I_{2}(q)\right)$. For a fixed $I(p+q)$, let $J(p+q)$ be an even permutation of $I(p+q)$ such that $j_{1}, \cdots, j_{p} \in I_{1}(p), j_{p+1}, \cdots, j_{p+q} \in$ $I_{2}(q)$. By denoting $J^{\prime}(q)=\left(j_{p+1}, \cdots, j_{p+q}\right)$ and using equation (1.8), from equation (3.12) we then obtain

$$
\begin{aligned}
\Theta_{I(p+q)}= & \frac{1}{(p+q) !} \sum_{\left(I_{1}, I_{2}\right)} \delta_{J(p)}^{I_{1}(p)} \Omega_{i_{11} i_{12}} \wedge \cdots \wedge \Omega_{i_{1, p-1} i_{1 p}} \\
& \wedge \delta_{J^{\prime}(q)}^{I_{2(q)}} \Omega_{i_{21} i_{22}} \wedge \cdots \wedge \Omega_{i_{2, q-1} j_{2 q}} \\
= & \frac{p ! q !}{(p+q) !} \sum_{\left(J, J^{\prime}\right)} \Theta_{J(p)} \wedge \Theta_{J^{\prime}(q)} .
\end{aligned}
$$

On the other hand, by the Laplace expansion of the determinant $A_{J(p+q), H(p+q)}$ according to the first $p$ rows we have

$$
\begin{aligned}
\sum_{H(p+q)} & A_{J(p+q), H(p+q)} \omega_{H(p+q)} \\
= & \sum_{H(p)} A_{J(p), H(q)} \omega_{H(p)} \wedge \sum_{H^{\prime}(q)} A_{J^{\prime}(q), H^{\prime}(q)} \omega_{H^{\prime}(q)}
\end{aligned}
$$

where $H^{\prime}(q)=\left(h_{p+1}, \cdots, h_{p+q}\right)$. Substituting equation (3.3) in equation (3.13) and using equation (3.14) we arrive at equation (3.11), and an application of Lemma 3.1 hence completes the proof of Lemma 3.5.

By repeatedly applying Lemma 3.5 we can easily obtain

Corollary 3.5.1. Let $p_{1}, \cdots, p_{k}$ be even positive integers, and $\left(m_{1}, \cdots, m_{k}\right)$ a k-tuple of nonnegative integers such that $q=\sum_{i=1}^{k} m_{i} p_{i} \leq n$. On a Riemannian manifold $M$ of dimension $n$, if condition (3.2) holds for $p_{1}, \cdots p_{k}$, then if also holds for $q$ with

$$
\kappa_{q}=\left(\kappa_{p_{1}}\right)^{m_{1} \cdots}\left(\kappa_{p_{k}}\right)^{m_{k}} \text {. }
$$

Corollary 3.5.2. On a Riemannian manifold $M$ of even dimension $n$ if condition (3.2) holds for some even $p$ dividing $n$, then

$$
\Theta_{1 \cdots n}=\left(\kappa_{p}\right)^{n / p}\left|a_{i j}\right| \omega_{1} \wedge \cdots \wedge \omega_{n}
$$

where $\omega_{1} \wedge \cdots \wedge \omega_{n}$ is the element of area of the manifold $M$. 
Combination of Theorem 2.1 with Corollary 3.5.2 gives immediately

Theorem 3.1. On a compact orientable Riemannian manifold $M$ of even dimension $n$ if condition (3.2) holds at every point $x$ for an even $p$ dividing $n$, and $(-1)^{n / 2}\left(\kappa_{p}\right)^{n / p}\left|a_{i j}\right|$ keeps a constant sign, then this sign is the sign of the Euler-Poincaré characteristic $\chi(M)$ of the manifold $M$. Moreover, under this hypothesis, $\chi(M)=0$ only when $\left(\kappa_{p}\right)^{n / p}\left|a_{i j}\right|$ vanishes identically.

This theorem was obtained by Chern [5] for $p=2$, and by Thorpe [8] for $a_{i j}=\delta_{i j}$.

For studying Pontrjagin classes we need

Lemma 3.6. Equation (3.3) can be written in the following form:

$$
\Theta_{I(p)}=\kappa_{p} \tilde{\omega}_{i_{1}} \wedge \cdots \wedge \tilde{\omega}_{i_{p}}
$$

where $\tilde{\omega}_{i_{\alpha}}$ are linear forms defined by

$$
\tilde{\omega}_{i_{\alpha}}=\sum_{h} a_{i_{\alpha} h} \omega_{h} \quad(\alpha=1, \cdots, p) .
$$

Proof. Let $p_{1}, p_{2}$ be any two positive integers such that $p_{1}+p_{2}=p$. Then by the Laplace theorem we can expand the determinant $A_{I(p), H(p)}$ according to the first $p_{1}$ row. By using this expansion it is easily seen that all $\left(\begin{array}{c}p \\ p_{1}\end{array}\right)$ terms of $\sum_{H(p)} A_{I(p), H(p)} \omega_{H(p)}$ are equal so that we have

$$
\begin{aligned}
\sum_{H(p)} A_{I(p), H(p)} \omega_{H(p)}=\frac{p !}{p_{1} ! p_{2} !} & \sum_{H\left(p_{1}\right)} A_{I\left(p_{1}\right), H\left(p_{1}\right)} \omega_{H\left(p_{1}\right)} \\
& \wedge \sum_{H^{\prime}\left(p_{2}\right)} A_{I^{\prime}\left(p_{2}\right), H^{\prime}\left(p_{2}\right)} \omega_{H^{\prime}\left(p_{2}\right)}
\end{aligned}
$$

where

$$
I^{\prime}\left(p_{2}\right)=\left(i_{p_{1}+1}, \cdots, i_{p}\right), \quad H^{\prime}\left(p_{2}\right)=\left(h_{p_{1}+1}, \cdots, h_{p}\right) .
$$

Repeatedly applying the same argument as above to both factors of the righthand side of equation (3.18) yields immediately equation (3.16).

Now we are in a position to prove the following theorem concerning the general curvature conditions for the vanishing of the Pontrjagin classes.

Theorem 3.2. On a compact orientable Riemannian manifold $M$ of dimension $n$ if condition (3.2) holds at every point $x$ for an even $p \leq n$, then the $k$-th Pontrjagin class $P_{k}(M)$ of the manifold $M$ is zero for all $k \geq p / 2$.

This theorem is due to Chern [5] for $p=2$, and to Thorpe [8] for $a_{i j}=\delta_{i j}$.

Proof. At first we consider the case $p \leq 2 k<2 p$. Let $\left(I_{1}(p), I_{2}(2 k-\right.$ $p)$ ) be a partition of a fixed $I(2 k)$, and $J(2 k)$ an even permutation 
of $I(2 k)$ such that $j_{1}, \cdots, j_{p} \in I_{1}(p), j_{p+1}, \cdots, j_{2 k} \in I_{2}(2 k-p)$. By denoting $J^{\prime}(2 k-p)=\left(j_{p+1}, \cdots, j_{2 k}\right)$, from equation (3.13) we have

$$
\Theta_{I(2 k)}=\frac{p !(2 k-p) !}{(2 k) !} \sum_{\left(J, J^{\prime}\right)} \Theta_{J(p)} \wedge \Theta_{J^{\prime}(2 k-p)},
$$

where $\sum_{\left(J, J^{\prime}\right)}$ denotes the summation over all such partitions of $I(2 k)$ into $\left(J(p), J^{\prime}(2 k-p)\right)$. By using condition (3.2) for $p$ and Lemmas 3.1 and 3.6 , equation (3.20) is reduced to

$$
\Theta_{I(2 k)}=\frac{p !(2 k-p) !}{(2 k) !} \kappa_{p} \sum_{\left(j, j^{\prime}\right)} \tilde{\omega}_{j_{1}} \wedge \cdots \wedge \tilde{\omega}_{j_{p}} \wedge \Theta_{J^{\prime}(2 k-p)}
$$

where $\tilde{\omega}_{j_{\alpha}}$ are linear forms defined by equation (3.17), so that $\Theta_{I(2 k)} \wedge$ $\Theta_{I(2 k)}$ is a sum, each term of which contains an exterior factor

$$
\tilde{\omega}_{j_{1}} \wedge \cdots \wedge \tilde{\omega}_{j_{p}} \wedge \tilde{\omega}_{j_{1}} \wedge \cdots \wedge \tilde{\omega}_{j_{p}}
$$

where each subscript $j \in I(2 k)$. Since $2 k<2 p$, at least two of these $j^{\prime} s$ must be equal, and therefore each of such factors (3.22) is zero. Thus $\Theta_{I(2 k)} \wedge \Theta_{I(2 k)}=0$ for all $I(2 k)$. By Theorem 2.2 we hence obtain $P_{k}(M)=0$ for all $k$ with $p / 2 \leq k<p$.

Since condition (3.2) is assumed to hold for $p$, by Corollary 3.5.1 it also holds for $2^{i} p(i=1,2, \cdots)$. Applying the same arguments as above we therefore have

$$
P_{k}(M)=0 \quad\left(2^{i-1} p \leq k<2^{i} p ; i=1,2, \cdots\right) .
$$

Hence $P_{k}(M)=0$ for all $k \geq p / 2$, and the theorem is proved.

\section{References}

[1] C. B. Allendoerfer, Global theorems in Riemannian geometry, Bull. Amer. Math. Soc. 54 (1948) 249-259.

[2] C. B. Allendoerfer \& A. Weil, The Gauss-Bonnet theorem for Riemannian polyhedra, Trans. Amer. Math. Soc. 53 (1943) 101-129.

[3] S. S. Chern, A simple intrinsic proof of the Gauss-Bonnet formula for closed Riemannian manifolds, Ann. of Math. 45 (1944) 747-752.

[4] - Topics in differential geometry, Mimeographed notes, Institute for Advanced Study, Princeton, 1951.

[5] - On curvature and characteristic classes of a Riemannian manifold, Adh. Math. Sem. Univ. Hamburg 20 (1956) 117-126.

[6] J. Eells, Jr., A generalization of the Gauss-Bonnet theorem, Trans. Amer. Math. Soc. 92 (1959) 142-153.

[7] F. D. Murnaghan, The generalized Kronecker symbol and its application to the theory of determinants, Amer. Math. Monthly 32 (1925) 233-241.

[8] J. A. Thorpe, Sectional curvatures and characteristic classes, Ann. of Math. 80 (1964) 429-443. 
